\title{
A Practical Apporach to Meter Placement in Distribution Networks
}

\author{
Bernd Brinkmann, Robert Scott, Karl Bicevskis and Michael Negnevitsky \\ School of Engineering and ICT \\ University of Tasmania \\ Hobart, Australia
}

\begin{abstract}
This paper presents a novel approach to the optimal meter placement problem in distribution networks. Conventional methods place meters in order to achieve a predefined level of accuracy for the estimated parameters such as voltages and currents. However, there is no generic method to determine this desired accuracy value for the individual parameters. Hence, a uniform accuracy value is generally chosen. Considering that parameters which are not in proximity to their constraint do not need to be monitored with a high level of accuracy, using a uniform accuracy for every estimated parameter may not be optimal. The method proposed in this paper takes a new approach which accounts for the proximity of the estimated parameters to their constraints. This approach can potentially reduce the number of required metering devices compared to conventional meter placement methods, while providing a practical level of state estimation accuracy.
\end{abstract}

Index Terms-Optimal meter placement, uncertainty, power system measurements, distribution network state estimation

\section{INTRODUCTION}

The amount of distributed generation such as wind and solar connected to distribution networks is expected to increase significantly in the future. Due to the uncertain and dynamic behavior of this type of generation, the currently passive distribution network has to become more active [1]. One of the main features of an active distribution network is the ability to react to changes in the operating state through near real-time monitoring and control of the system. For this to be effective, the state of the network has to be available in near real-time with a sufficient degree of accuracy. However, most distribution networks only have a limited number of measurements. This can result in a significant amount of uncertainty in the estimated state. Hence, additional metering devices are often required in order to increase the state estimation accuracy. Due to economic constraints, however, only a limited number of metering devices can be placed in the network. This leads to the problem of optimal meter placement.

In transmission networks, metering devices are placed in order to achieve observability or to improve redundancy in terms of measurements. In distribution networks, however, observability is achieved by the use of pseudo-measurements which generally make up a large proportion of total measurements. Pseudo-measurements are forecasts for loads and distributed generation based on historical data. Since it is not possible to accurately predict loads and distributed generation from historical data, pseudo-measurements generally have large margins of error associated with them. This means that the estimated state can contain a significant amount of uncertainty. For this reason, the objective of a meter placement in distribution networks is to increase the accuracy of the state estimation result to a desired level [1-3]. However, no generic method exists to determine the desired level of accuracy for the individual estimated parameters. Hence, a uniform desired level of accuracy is generally defined for the estimated parameters. While this approach can ensure that a sufficient degree of accuracy is achieved throughout the network, some parameters which are not close to their constraints may be estimated with a higher degree of accuracy than required. Therefore, the resulting meter placement might not be optimal. The constraints of a network are defined by regulations (e.g. the voltage compliance range) and its physical properties (e.g. thermal limits of network components) [4]. In [5] a new meter placement method has been proposed that increases the accuracy of the estimated parameters depending upon their proximity to their respective constraints under worst case considerations. Here worst case scenarios refer to the network states in which the constraints are most likely to be violated. Hence, the required accuracy is determined for each parameter individually. As a result, parameters that are not in proximity of their constraint under worst case consideration do not have to be estimated with the same degree of accuracy as parameters that are within constraint proximity. However, this approach has the limitation that a large number of measurements may have to be placed if a parameter is very close to a constraint.

In order to address these limitations, this paper proposes a meter placement method which uses a combination of the method proposed in [5] and the conventional meter placement approach. The proposed method increases the accuracy of the estimated parameters depending upon their proximity to their constraint. If, however, the accuracy of an estimated parameter is equal to or higher than a desired value, no additional 
metering devices will be placed in order to further increase the accuracy of this particular parameter. This approach addresses the limitation of both methods mentioned above.

The rest of this paper is structured as follows. In Section II the two different approaches to optimal meter placement in distribution networks, which are used in this paper, are explained. Section III outlines the proposed approach, and a case study is provided in Section IV. Finally, a conclusion is drawn in Section V.

\section{EXISTING Meter Placement Methods}

\section{A. Conventional meter placement approach}

To enhance the accuracy of state estimation, numerous meter placement methods are proposed in the literature [1-3, $6,7]$. As mentioned in Section I, these methods generally aim to improve the accuracy of the estimated parameters until a desired accuracy threshold is met. Hence, meters are added until all estimated parameters satisfy a predefined accuracy threshold. The accuracy of an estimated parameter is calculated from the relative difference between an estimate obtained with and without considering the influence of measurement noise.

$$
\frac{\left|\hat{E}_{i}-E_{i}\right|}{E_{i}} \leq t h
$$

where $i=[1,2, \ldots, i, \ldots, N], \quad N$ is the total number of estimated parameters, $E_{i}$ is the value of the estimated parameter $i$ calculated without measurement noise, $\hat{E}_{i}$ is the value of the $i^{\text {th }}$ estimated parameter calculated with measurement noise, and th is the desired accuracy threshold.

\section{B. Individually determined accuracy recuirement}

The proposed approach in [5] determines the required state estimation accuracy by assessing the proximity of the estimated parameters to their constraints under worst case consideration. In this approach the accuracy of an estimated parameter has to be at least high enough to determine if an estimated parameter is within its constraints under normal operating conditions. A network is in its normal operating state if no constraints are violated and all connected loads are supplied with electric power [8]. Hence, the required accuracy for the estimated parameter $i$ is calculated by

$$
\begin{aligned}
R A_{i, \min } & =-\left[i_{\min }-\hat{E}_{i}\right] \\
R A_{i, \max } & =\left[i_{\max }-\hat{E}_{i}\right]
\end{aligned}
$$

where the lower and upper constraints for the estimated parameter $i$ are represented by $i_{\text {min }}$ and $i_{\max }$ respectively, and the required accuracy for the parameter $i$ is given by $R A_{i, \min }$ and $R A_{i, \max }$ for values below and above $\hat{E}_{i}$ respectively.

The objective of the meter placement is to increase the accuracy until the following criterion is satisfied for every estimated parameter

$$
a_{i, \min } \geq R A_{i, \min } \wedge a_{i, \max } \leq R A_{i, \max }
$$

where $a_{i, \min }$ and $a_{i, \max }$ are the endpoints of the confidence interval (CI) of the $i^{\text {th }}$ estimated parameter. These endpoints represent the maximum expected difference between the estimate and its real value, and therefore, provide the accuracy of the estimate.

To calculate $a_{i, \min }$ and $a_{i, \max }$ the probability density function (PDF) of the $i^{\text {th }}$ estimated parameter has to be obtained first. For this purpose a number of methods are available in literature [9-12]. Since the PDF is a continuous function which ranges from plus infinity to negative infinity, a confidence level (CL) has to be applied. The CL specifies the confidence that the real value is between $a_{i, \min }$ and $a_{i, \max }$. Hence, the endpoints of the CI have to satisfy the following equations

$$
\begin{aligned}
& \int_{a_{i, \min }}^{\hat{E}_{i}} P D F_{i}\left(a_{i}\right) d a_{i}=C L / 2 \\
& a_{i, \max } \\
& \int_{\hat{E}_{i}} P D F_{i}\left(a_{i}\right) d a_{i}=C L / 2
\end{aligned}
$$

The confidence interval can be calculated by multiplying the standard deviation of the estimate with a coverage factor if the estimation error is Gaussian distributed. If the state estimation error is not Gaussian distributed, other well-known methods such as bootstrapping can be used [13].

\section{PROPOSED METER PLACEMENT Method}

The proposed meter placement method combines the conventional approach of using a desired minimum accuracy (1), to the approach outlined in Section II, B, which uses individually determined accuracy requirements (4). Therefore, the proposed approach aims to place metering devices until every estimated parameter fulfills either (1) or (4). As a result, the limitations stated in Section I can be addressed.

\section{A. Compliance ratio}

Due to the random nature of the measurement noise, it is always possible that an estimated parameter does not satisfy (1) or (4). No matter how many measurements are placed in a network. For this reason, a compliance ratio $(C R)$ is used that determines how likely an estimated parameter satisfies (4) or (1). This CR is calculated by performing a predefined number of Monte Carlo simulations. In each simulation, a set of state estimation inputs are generated according to their predefined measurement accuracies. The state of the network is then estimated for each generated set of state estimation inputs. After a sufficient number of simulations, the CR of an estimated parameter is calculated as the ratio of estimates that fulfilled (1) or (4) by the total number of simulations. If CR is equal to or greater than a predefined value, for instance $99 \%$, the parameter is considered compliant. Hence, additional metering devices are placed until the CR of all estimated parameters is equal to or greater than a predefined value for each worst case scenario. 


\section{B. Worst Case Scenarios}

The accuracy of the state estimation changes with the network state. Hence, all possible network states would have to be assessed. However, since the proposed method focuses on the probability that the constraints of the network are violated, it is only required to assess the network state where the constraints are most likely to be violated. Thus, only the worst case scenarios are considered. These scenarios are used for network planning purposes and should, therefore, be available to the network operator [14-16]. Generally two cases have to be assessed, maximum load/minimum generation, and minimum load/maximum distributed generation [17].

\section{Meter placement algorithm}

The following steps describe the proposed method in detail:

Step 1: Define the desired compliance ratio (CR), confidence level (CL), number of simulations $\left(N_{\text {num }}\right)$ and the desired level of accuracy $(t h)$. Go to Step 2.

Step 2: Select a worst case scenario which has not yet been evaluated. Go to Step 3.

Step 3: Set the current simulation count to one $(\mathrm{N}=1)$. Go to Step 4.

Step 4: Generate a set of random SE inputs according to their respective predefined measurement accuracies. Go to Step 5.

Step 5: Estimate the network state. Go to Step 6.

Step 6: Calculate the PDF of each estimated parameter. Go to Step 7.

Step 7: Calculate the confidence interval $(C I)$ for all estimated parameters as discussed in Section II, B. Go to Step 8.

Step 8: Calculate the required accuracy using Equations (2) and (3). Go to Step 9.

Step 9: Determine the estimated parameters which satisfy (1) or (4). Go to Step 10.

Step 10: Record the compliant parameters identified in Step 9 and increase the current simulation number by one $(\mathrm{N}=\mathrm{N}+1)$. Go to Step 11 .

Step 11: Determine whether the specified number of simulations has been met $\left(\mathrm{N}>\mathrm{N}_{\text {num }}\right)$. If yes, go to Step 12. If no, return to Step 4.

Step 12: Determine whether all worst case scenarios have been evaluated. If yes, go to Step 13. If no, go to Step 2.

Step 13: Calculate the compliance ratio for each estimated parameter and each worst case scenario. Go to Step 14.

Step 14: Do all estimated parameters meet the desired compliance ratio for each worst case scenario? If yes, stop. If not, place an additional metering device according to the method detailed in Section III, D and return to Step 2.

\section{Selection of Metering position}

In the case that not all estimated parameters satisfy the $\mathrm{CR}$, additional metering devices are placed one meter at a time. This ensures that the computation time does not increase exponentially with the number of required metering devices. In order to find the best position for a metering device, every possible location is assessed. The position which results in the highest number of parameters that satisfy (4) or (1) for each worst case scenario with a predefined compliance ratio, is chosen as the best location for an additional metering device. If, however, more than one meter placement results in the same improvement in the number of compliant parameters, the best position is determined by an uncertainty index (UI) [18]. This index gives an indication of the uncertainty in the state estimation result by using information entropy. The UI is calculated from the PDFs of the estimated parameters. In order to calculate the UI, the infinite number of possible values for the estimated parameters has to be reduced to a finite number. This is achieved by "binning" the estimated parameters. Each "bin" represents a range of possible values of the $i^{t h}$ estimated parameter. The bin size has to be chosen small enough to accurately represent the PDF of the estimated parameters. The UI is calculated by

$$
U I=\sum_{i=1}^{N} X_{i}
$$

where $N$ represents the total number of estimated parameters, and $X_{i}$ is the entropy of the parameter $i$.

$X_{i}$ is defined by

$$
X_{i}=-\sum_{k=1}^{B_{i}} P_{i}\left(b_{i}^{k}\right) \log _{2} P_{i}\left(b_{i}^{k}\right),
$$

where $b_{i}^{k}$ represents the range of possible values within a particular bin, $k$, for the estimated parameter $i$, the total number of bins for the $i^{\text {th }}$ estimated parameter is given by $B_{i}$, and the probability that the true value of parameter $i$ is within $b_{i}^{k}$ is given by $P_{i}\left(b_{i}^{k}\right)$.

The probability that the true value of parameter $i$ is within a particular bin, $b_{i}^{k}$, is given by the area under the PDF within bin $b_{i}^{k}$. The UI has to be calculated for each worst case scenario and each potential metering configuration that has to be compared. The highest UI from the worst case scenarios is used to compare the potential metering positions with each other. Out of this set of UIs the lowest value is chosen as the best metering position since a low UI relates to a low uncertainty in the state estimation result

$$
\min _{1 \leq n \leq N}\left[\max _{1 \leq c \leq C}\left[U I_{c, n}\right]\right]
$$

where the potential meter placements are represented by $n$ for $n=[1,2, \ldots, n \ldots, M]$, The total number of potential meter placements are given by $M, c$ is a worst case scenario for $c=[1,2, \ldots, c, \ldots, C] ; \mathrm{C}$ is the total number of worst case 
scenarios, and $U I_{c, n}$ is the uncertainty index for worst case scenario $c$ and meter placement $n$.

The following steps describe how the best metering position is found.

Step 1: Determine the number of busses which meet the desired compliance ratio as described in Section III, C. Go to Step 2.

Step 2: Find the meter placement which results in the highest number of busses that meet the compliance ratio for both worst case scenarios. Go to Step 3.

Step 3: Does more than one potential metering location result in the highest number of compliant busses? If no, place a metering device at that bus and stop. If yes, go to Step 4.

Step 4: Calculate the UI for every worst case scenario and metering configuration which result in the highest number of compliant busses. Go to Step 5 .

Step 5: Find the best position for the additional metering device by using (15).

\section{CASE STUdy}

The proposed methodology is applied to two case studies. The first case study is conducted on a simple 7-Bus radial test feeder. The second case study is performed on a modified IEEE 34-Bus test network. For simplicity balanced conditions are assumed in both case studies.

The following assumptions are made:

- All measurement errors are normally distributed.

- Metering devices have the measurement accuracy of $\pm 1 \%$.

- All network loads are represented by pseudomeasurements, with the measurement accuracy of $\pm 50 \%$.

- Distributed generation is proportional to the load connected at each bus and is represented by pseudomeasurements, with the measurement accuracy of $\pm 100 \%$.

Since measurement errors are assumed to be normally distributed, the standard deviation can be calculated by the product of one-third of the measurement accuracy and the expected value [5]. The case studies focus on improving the accuracy of the voltage estimate.

\section{A. 7-Bus Radial Test Feeder}

The 7-Bus feeder is shown in Fig. 1; where a voltage meter is located at bus 1, all lines are of equal length, and the loads connected at each bus are of equal value.

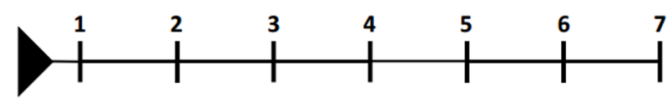

Fig. 1. 7-Bus Test Feeder.

This case study considers two worst case scenarios. The first case is maximum load and no distributed generation (worst case 1). The second case (worst case 2) is maximum distributed generation (equal to $20 \%$ of the maximum load at each bus) and minimum load (equal to $30 \%$ of the maximum load at each bus). The estimated voltage profile for each worst case scenario is shown in Figs 2 and 3.

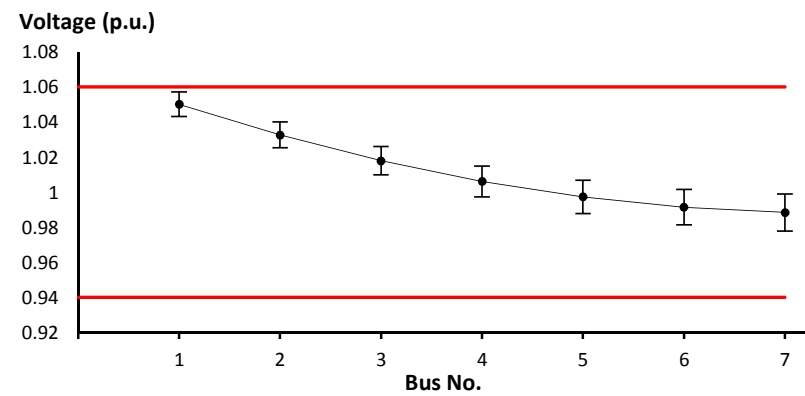

Fig. 2. Voltage profile for the 7-Bus feeder under maximum load and no distributed generation.

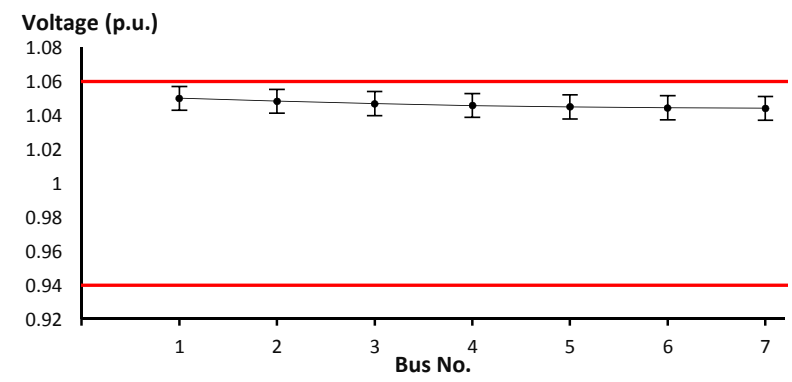

Fig. 3. Voltage profile for the 7-Bus feeder under minimum load and maximum distributed generation.

In Figs 2 and 3, the error bars represent the 95\% CI of the estimated voltage. The bars at 0.94 p.u. and 1.06 p.u. represent the voltage constraints which are set according to the Tasmanian Electricity Code [19]. The desired accuracy threshold th is set to $1 \%$, the CL is set to $95 \%$ and the desired $\mathrm{CR}$ is set to $99 \%$. These values are for demonstration purposes only, and for real networks, these levels must be specified by the network operator depending upon the individual application.

To determine the CR of an estimated parameter, its $P D F$ needs to be calculated first. This has been done by using the gain matrix to obtain the standard deviations of the estimated parameters [19]. The CR is then calculated through Monte Carlo simulations. A sufficiently large number of simulations are necessary to ensure that $\mathrm{CR}$ convergences to its final value. In this case study, 500 simulations are used.

The resulting CRs for both worst case scenarios are shown in Table I. In this table, Criterion 1 refers to the CRs calculated when only (1) is considered; Criterion 2 refers to 
the CRs calculated when only (4) is considered; and Criterion 1 or 2 refers to the proposed method where either (1) or (4) has to be satisfied.

Table I. Compliance ratios with single meter at bus 1

\begin{tabular}{|c|c|c|c|c|c|c|}
\hline & \multicolumn{3}{|c|}{$\begin{array}{l}\text { Maximum load and no } \\
\text { distributed generation }\end{array}$} & \multicolumn{3}{|c|}{$\begin{array}{l}\text { Minimum load and maximum } \\
\text { distributed generation }\end{array}$} \\
\hline $\begin{array}{l}\text { Bus } \\
\text { No. }\end{array}$ & Crit. 1 & Crit. 2 & $\begin{array}{l}\text { Crit. } \\
1 \text { or } 2\end{array}$ & Crit. 1 & Crit. 2 & $\begin{array}{l}\text { Crit. } \\
1 \text { or } 2\end{array}$ \\
\hline 1 & $99.7 \%$ & $81.3 \%$ & $99.8 \%$ & $99.6 \%$ & $82.0 \%$ & $99.9 \%$ \\
\hline 2 & $99.6 \%$ & $100.0 \%$ & $100.0 \%$ & $99.6 \%$ & $91.5 \%$ & $99.9 \%$ \\
\hline 3 & $99.0 \%$ & $100.0 \%$ & $100.0 \%$ & $99.5 \%$ & $95.5 \%$ & $99.9 \%$ \\
\hline 4 & $97.8 \%$ & $100.0 \%$ & $100.0 \%$ & $99.5 \%$ & $97.1 \%$ & $99.9 \%$ \\
\hline 5 & $96.3 \%$ & $100.0 \%$ & $100.0 \%$ & $99.3 \%$ & $97.9 \%$ & $99.8 \%$ \\
\hline 6 & $95.0 \%$ & $100.0 \%$ & $100.0 \%$ & $99.2 \%$ & $98.2 \%$ & $99.8 \%$ \\
\hline 7 & $94.1 \%$ & $100.0 \%$ & $100.0 \%$ & $99.2 \%$ & $98.3 \%$ & $99.8 \%$ \\
\hline
\end{tabular}

As can be observed, if the CRs are determined using either criterion 1 or criterion 2 individually, neither assessment meets the desired $99 \% \mathrm{CR}$ for both worst case scenarios and all estimated parameters. Thus, using either criterion individually would require the placement of an additional meter to improve the state estimation accuracy. However, by using the proposed method where either (1) or (4) has to be satisfied, all parameters meet the required 99\% $C R$, and no additional meters are necessary. This clearly demonstrates the advantage of the proposed method, where measurement devices are only placed to improve the estimation accuracy of critical parameters while avoiding unnecessarily high levels of accuracy.

\section{B. IEEE Test Feeder}

To further illustrate the proposed method a second case study is performed on a modified 34-Bus IEEE test network. A one-line diagram of this network is shown in Fig. 4; with the original data available in [20]. For simplicity, all voltage control devices are removed. To maintain normal network operating conditions, all network loads are reduced by a factor of three.

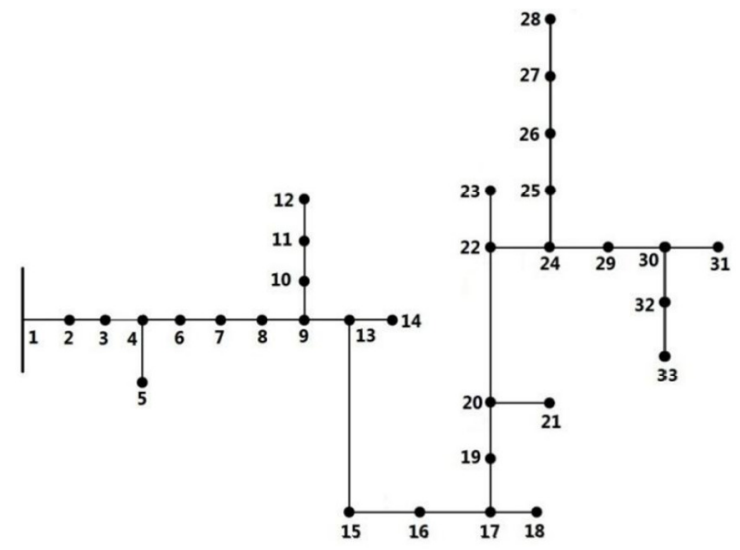

Fig. 4. Modified IEEE 34-bus test feeder

It is assumed that a voltage meter is located at Bus 1 only. The two worst case scenarios previously considered are also adapted for use in this case study. The estimated voltage profile of each worst case scenario is shown in Figs 5 and 6. The bars represent the 95\% CI.

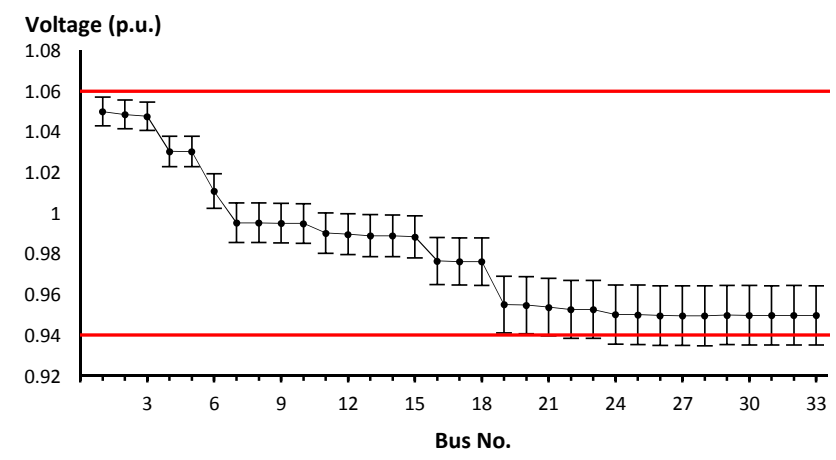

Fig. 5. Voltage profile for maximum load and no distributed generation.

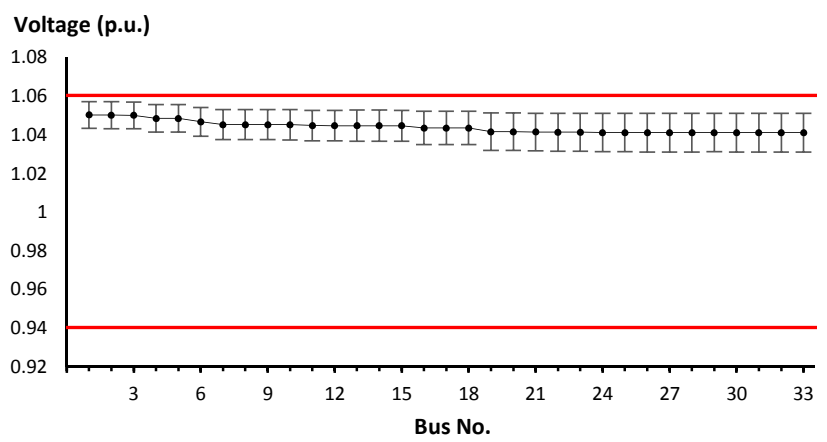

Fig. 6. Voltage profile for maximum load and no distributed generation.

The $C R$ for each network parameter has been calculated for each worst case scenario and the results are shown in Table II.

Table II. Compliance ratios with single meter at bus 1 (case study 2)

\begin{tabular}{|c|c|c|c|c|c|}
\hline $\begin{array}{l}\text { Bus } \\
\text { No. }\end{array}$ & $\begin{array}{c}\text { Worst } \\
\text { Case } 1\end{array}$ & $\begin{array}{r}\text { Worst } \\
\text { Case } 2 \\
\end{array}$ & $\begin{array}{l}\text { Bus } \\
\text { No. }\end{array}$ & $\begin{array}{c}\text { Worst Case } \\
1\end{array}$ & $\begin{array}{c}\text { Worst Case } \\
2\end{array}$ \\
\hline 1 & $100.0 \%$ & $99.9 \%$ & 18 & $100.0 \%$ & $99.6 \%$ \\
\hline 2 & $100.0 \%$ & $99.9 \%$ & 19 & $90.5 \%$ & $99.3 \%$ \\
\hline 3 & $100.0 \%$ & $99.9 \%$ & 20 & $90.5 \%$ & $99.3 \%$ \\
\hline 4 & $100.0 \%$ & $99.9 \%$ & 21 & $90.2 \%$ & $99.3 \%$ \\
\hline 5 & $100.0 \%$ & $99.9 \%$ & 22 & $89.9 \%$ & $99.3 \%$ \\
\hline 6 & $100.0 \%$ & $99.9 \%$ & 23 & $89.9 \%$ & $99.3 \%$ \\
\hline 7 & $100.0 \%$ & $99.9 \%$ & 24 & $89.3 \%$ & $99.2 \%$ \\
\hline 8 & $100.0 \%$ & $99.9 \%$ & 25 & $89.3 \%$ & $99.2 \%$ \\
\hline 9 & $100.0 \%$ & $99.9 \%$ & 26 & $89.1 \%$ & $99.2 \%$ \\
\hline 10 & $100.0 \%$ & $99.9 \%$ & 27 & $89.1 \%$ & $99.2 \%$ \\
\hline 11 & $100.0 \%$ & $99.8 \%$ & 28 & $89.1 \%$ & $99.2 \%$ \\
\hline 12 & $100.0 \%$ & $99.8 \%$ & 29 & $89.3 \%$ & $99.2 \%$ \\
\hline 13 & $100.0 \%$ & $99.8 \%$ & 30 & $89.3 \%$ & $99.2 \%$ \\
\hline 14 & $100.0 \%$ & $99.8 \%$ & 31 & $89.3 \%$ & $99.2 \%$ \\
\hline 15 & $100.0 \%$ & $99.8 \%$ & 32 & $89.3 \%$ & $99.2 \%$ \\
\hline 16 & $100.0 \%$ & $99.6 \%$ & 33 & $89.3 \%$ & $99.2 \%$ \\
\hline 17 & $100.0 \%$ & $99.6 \%$ & & & \\
\hline
\end{tabular}

From Table II, it can be seen that estimated parameters at buses 19-33 did not meet the desired CR of $99 \%$ for both 
worst case scenarios. Therefore, the state estimation accuracy is not sufficient. To improve the accuracy of the state estimation, it is necessary that additional measurement devices are positioned in the network. By sequentially placing a meter at each bus and checking how many estimated parameters meet the required 99\% CR, it was determined that an additional meter placement at any bus 2033 results in all network parameters meeting the minimum compliancy requirement. The optimal metering location is thus determined by evaluating which potential metering location results in the lowest UI, according to (15). This determined the best metering location to be at bus 30 . With this single additional meter placement at bus 30, all estimated parameters for each worst case scenario now satisfy the required $99 \% C R$.

\section{CONCLUSION}

A new approach to optimal meter placement in distribution networks has been proposed in this paper. This method places additional metering devices until it is possible to determine if the estimated parameters are within their constraints under normal operating conditions, or a predefined minimum accuracy is achieved. The proposed method is based on the fact that parameters which are not in proximity to their constraints are not required to be known with a high degree of accuracy. Hence, only parameters which are within proximity or outside of their constraints have to be monitored closely. As a result, a reduced number of additional metering devices may be required compared to conventional methods, while maintaining a practical level of state estimation accuracy. The advantages and practicality of the proposed method were demonstrated in two case studies on a simple 7-Bus network and a modified 34-Bus IEEE test feeder.

\section{REFERENCES}

[1] L. Junqi, F. Ponci, A. Monti, C. Muscas, P. A. Pegoraro, and S. Sulis, "Optimal Meter Placement for Robust Measurement Systems in Active Distribution Grids," IEEE Trans. Instrum. Meas., vol. 63, no. 5, pp. 1096-1105, 2014.

[2] N. Nusrat, M. Irving, and G. Taylor, "Novel meter placement algorithm for enhanced accuracy of distribution system state estimation," in Proc. IEEE Power and Energy Society General Meeting, San Diego, CA 2012, pp. 1-8.

[3] R. Singh, B. C. Pal, R. A. Jabr, and R. B. Vinter, "Meter Placement for Distribution System State Estimation: An Ordinal Optimization Approach," IEEE Transactions on Power Systems, vol. 26, no. 4, pp. 2328-2335, 2011
[4] B. Brinkmann and M. Negnevitsky, "A Practical Approach to Observability Analysis and State Estimation in Distribution Networks," in Proc. IEEE Power and Energy Society General Meeting, Boston, MA, 2016, pp. 1-5.

[5] B. Brinkmann and M. Negnevitsky, "A Probabilistic Approach to Observability of Distribution Networks," IEEE Trans. on Power Syst., pp. 1-10, 2016.

[6] D. Echternacht and A. Moser, "Cost optimal meter placement in low and medium voltagte grids considering stochastic dependencies," in Proc. Innovative Smart Grid Technologies Europe (ISGT EUROPE), 2013, pp. 1-5.

[7] J. Liu, J. Tang, F. Ponci, A. Monti, C. Muscas, and P. A. Pegoraro, "Trade-Offs in PMU Deployment for State Estimation in Active Distribution Grids," IEEE Transactions on Smart Grid, vol. 3, no. 2, pp. 915-924, 2012.

[8] A. Abur and A. G. Expósito, Power System State Estimation Theory and Implementation. New York: Marcel Dekker, Inc., 2004.

[9] M. Strelec, P. Janecek, D. Georgiev, A. Zapotocka, and E. Janecek, "Backward/forward probabilistic network state estimation tool and its real world validation," in Proc. 56th International Scientific Conference on Power and Electrical Engineering of Riga Technical University (RTUCON), 2015, pp. 1-6.

[10] A. K. Ghosh, D. L. Lubkeman, M. J. Downey, and R. H. Jones, "Distribution circuit state estimation using a probabilistic approach," IEEE Trans. on Power Syst., vol. 12, no. 1, pp. 45-51, 1997.

[11] G. Valverde, A. T. Saric, and V. Terzija, "Stochastic Monitoring of Distribution Networks Including Correlated Input Variables," IEEE Trans. on Power Syst., vol. 28, no. 1, pp. 246-255, 2013.

[12] L. Guang, Z. Ning, T. Ferryman, and F. Tuffner, "Uncertainty quantification in state estimation using the probabilistic collocation method," in Proc. IEEE/PES Power Systems Conference and Exposition (PSCE), 2011, pp. 1-8.

[13] M. J. Crawley, Statistics an introduction using $R$. UK: John Wiley \& Sons, Inc., 2005.

[14] A. Kulmala, S. Repo, and P. Jarventausta, "Using statistical distribution network planning for voltage control method selection," in Proc. Renewable Power Generation Conf., Edinburgh, 2011, pp. 1-6.

[15] M. O. W. Grond, J. Morren, and J. G. Slootweg, "Integrating smart grid solutions into distribution network planning," in Proc. PowerTech (POWERTECH), 2013 IEEE Grenoble, 2013, pp. 1-6.

[16] G. Celli, F. Pilo, G. G. Soma, R. Cicoria, G. Mauri, E. Fasciolo, et al., "A comparison of distribution network planning solutions: Traditional reinforcement versus integration of distributed energy storage," in Proc. IEEE PowerTech (POWERTECH) Grenoble, 2013, pp. 1-6.

[17] M. O. W. Grond, H. N. Luong, J. Morren, P. A. N. Bosman, H. J. G. Slootweg, and H. La Poutre, "Practice-oriented optimization of distribution network planning using metaheuristic algorithms," in Proc. Power Syst. Computation Conf. (PSCC), Wroclaw, 2014, pp. 1-8.

[18] B. Brinkmann, M. Negnevitsky, T. Yee, and T. Nguyen, "An Observability Index for Distribution Networks using Information Entropy," in Proc. Australasian Universities Power Engineering Conf. (AUPEC), Wollongong, NSW, 2015, pp. 1-6.

[19] (1 July 2014). Tasmanian Electricity Code. Available: http://www.energyregulator.tas.gov.au/domino/otter.nsf/elect-v/003

[20] (05 August 2013). IEEE PES Radial Distribution Test Feeders Available: http://ewh.ieee.org/soc/pes/dsacom/testfeeders/ 